Acta Crystallographica Section D

Biological Crystallography

ISSN 1399-0047

\title{
Structural basis for the broad specificity of a new family of amino-acid racemases
}

Broad-spectrum amino-acid racemases (Bsrs) enable bacteria to generate noncanonical D-amino acids, the roles of which in microbial physiology, including the modulation of cell-wall structure and the dissolution of biofilms, are just beginning to be appreciated. Here, extensive crystallographic, mutational, biochemical and bioinformatic studies were used to define the molecular features of the racemase BsrV that enable this enzyme to accommodate more diverse substrates than the related PLP-dependent alanine racemases. Conserved residues were identified that distinguish BsrV and a newly defined family of broad-spectrum racemases from alanine racemases, and these residues were found to be key mediators of the multispecificity of BrsV. Finally, the structural analysis of an additional Bsr that was identified in the bioinformatic analysis confirmed that the distinguishing features of $\mathrm{BrsV}$ are conserved among Bsr family members.

\section{Introduction}

In nature, the majority of D-amino acids (DAA) are found in bacterial cell walls, where D-Ala and D-Glu are key constituents of the short peptides that cross-link the glycan chains within the peptidoglycan (PG) polymer (Vollmer et al., 2008). These DAA are produced from their corresponding L-enantiomers by highly specific alanine and glutamate amino-acid racemases (Alr and MurI), which have distinct structural and catalytic properties (Cava, Lam et al., 2011). Alr (EC 5.1.1.1), which is the best characterized of these enzymes, is a cytoplasmic protein that requires the ubiquitous coenzyme pyroxidal-5-phosphate (PLP) as a cofactor (Richard et al., 2009). Crystallographic studies of Alr from diverse organisms have enabled the identification of the structural determinants and spatial constrains that account for its mono-specificity (Couñago et al., 2009; LeMagueres et al., 2003, 2005; Noda et al., 2004; Shaw et al., 1997; Strych \& Benedik, 2002; Wu et al., 2008).

Recently, we demonstrated that diverse bacteria produce and release to the extracellular medium high amounts of DAA that are not canonical components of the cell wall (e.g. D-Ala and D-Glu; Cava, de Pedro et al., 2011; Lam et al., 2009). In Vibrio cholerae, the production of these noncanonical DAA (NCDAA) is dependent upon BsrV (VC1312), a periplasmic broad-spectrum racemase (Bsr) with high sequence homology ( $E$-value of $1 \times 10^{-25}, 28 \%$ identity) to the primary alanine racemase of $V$. cholerae, AlrV (VC0372; Fig. $1 a$ and Supplementary Fig. S1a $a^{\mathbf{1}}$ Lam et al., 2009). A subset of Alr-related racemases from other organisms have also been reported to accept multiple amino-acid substrates (Kino et al., 2007; Lam

\footnotetext{
${ }^{1}$ Supporting information has been deposited in the IUCr electronic archive (Reference: DW5071).
}

Received 2 August 2013

Accepted 5 September 2013

PDB References: BsrV, 4beu; $\triangle \mathrm{Cl}$-BsrV, 4beq; $\mathrm{Bsr}_{\mathrm{Ah}}$, 4bf5; $\mathrm{Alr}_{\text {Ah, }}$ 4bhy 
et al., 2009; Matsui \& Oikawa, 2010; Wu et al., 2008). In $V$. cholerae, the presence of NCDAA negatively regulates cellwall metabolism, at least in part owing to their incoporation into PG (Cava, de Pedro et al., 2011; Cava, Lam et al., 2011; Lam et al., 2009). NCDAA have also been reported to influence biofilm stability (Kolodkin-Gal et al., 2010), virulence (Anfora et al., 2007) and sporulation (O'Connor \& Zusman, 1997). Susceptibility to NCDAA seems to be more widespread than production of NCDAA, raising the possibility that some bacteria may produce and release NCDAA in order to influence nonproducing species within the same habitat (Cava, de Pedro et al., 2011; Horcajo et al., 2012). Given the important
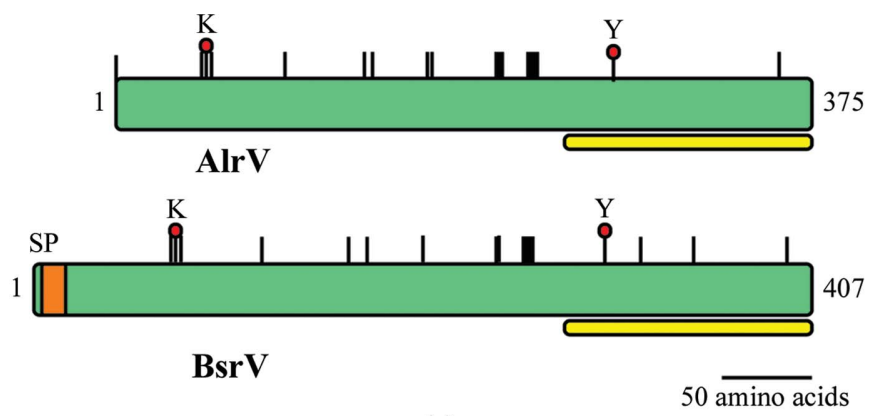

(a)

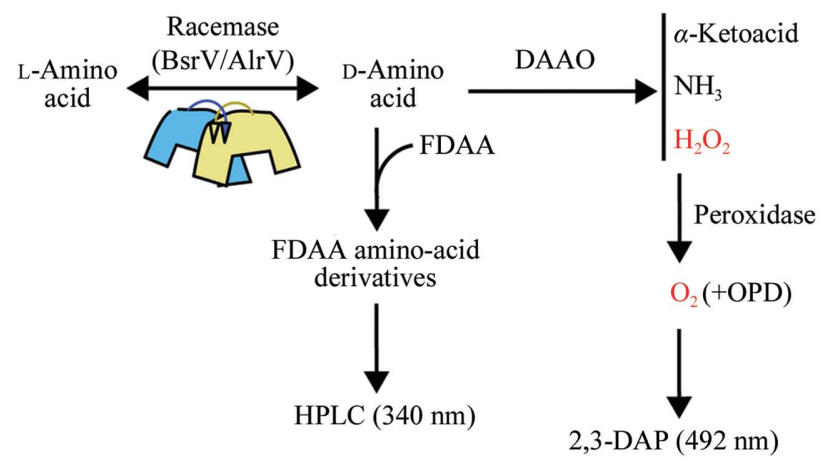

(b)

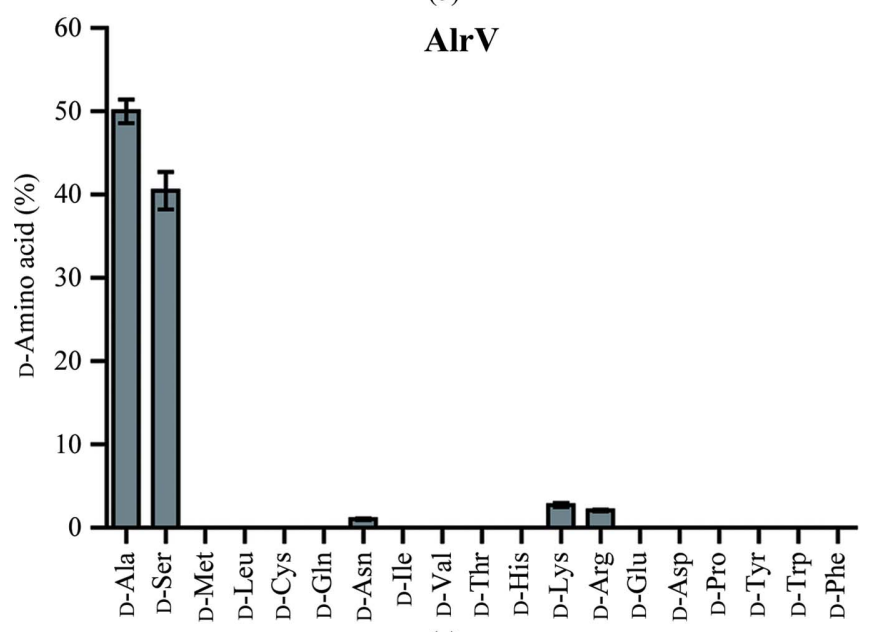

(c)

Figure 1

Biochemical comparison of AlrV and BsrV from V. cholerae. (a) Predicted protein features of the monospecific alanine racemase (AlrV) and broadspectrum racemase (BsrV) from $V$. cholerae generated with the NCBI protein-analysis package. Dimerization motifs are in yellow. SP, signal peptide. Red circles pinpoint the catalytic Lys and Tyr residues. Vertical lines correspond to conserved functional residues (PLP binding, substrate binding, catalytic site and dimeric interface). (b) Schematic of the different methods used to assess amino-acid racemase activities (see $\$ 2$ ). ( $c, d)$ D-Amino-acid accumulation in the presence of $\operatorname{AlrV}(c)$ and $\operatorname{BsrV}(d)$ after 90 min reactions with various substrates revealed through Marfey's derivatization. The results in $(c)$ and $(d)$ are the means \pm SD of triplicates from two independent experiments. See also Supplementary Fig. S1. adaptative properties provided by NCDAA, a detailed investigation of multi-specific racemases will be critical to understand NCDAA-controlled processes and for the development of new drugs to combact pathogenic bacteria (Conti et al., 2011).

Here, we explore the capacity of BsrV to utilize a wide variety of substrates using a combination of structural, biochemical, mutational and bioinformatic approaches. Finally, we also define and experimentally validate a molecular footprint of conserved residues that mediate the multispecificity of Brs V and use this signature sequence to identify a large family of BsrV-like multi-specific racemases in bacteria.

\section{Materials and methods}

\subsection{Microbiology}

All $V$. cholerae strains used in this study were derived from the sequenced El Tor clinical isolate N16961 (Heidelberg et al., 2000). Plasmids and primers, growth conditions and standard molecular-biology techniques are described in the Supporting Information.

\subsection{Racemase activity assays}

All racemase assays were performed in two fundamental steps: (i) the racemase reaction and (ii) the DAAO reaction coupled to peroxidase and 2,3-diaminophenazine or Marfey's (FDAA) derivatization (Fig. 1b). The formation of the colorimetric product was measured at $492 \mathrm{~nm}$ (see Supporting Information). The steady-state kinetics parameters were determined using Microsoft Office 2007 and Solver Macro (De Levie, 2001; see Supporting Information). For competition assays, FDAA derivatization of $\mathrm{L}$ and $\mathrm{D}$ forms of amino acids

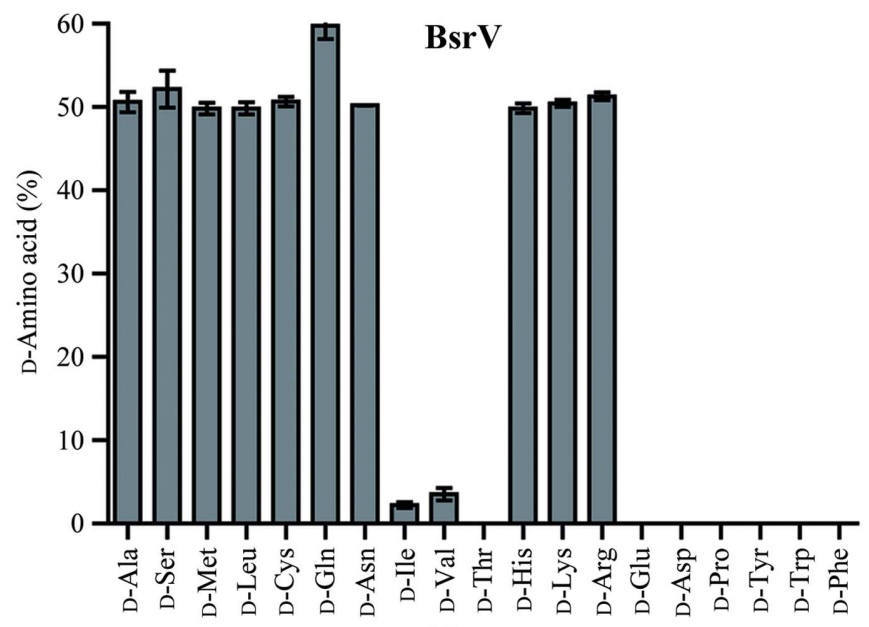

(d) 
Table 1

Kinetic parameters $\left(K_{\mathrm{m}}, k_{\mathrm{cat}}\right.$ and $\left.k_{\mathrm{cat}} / K_{\mathrm{m}}\right)$ of $\mathrm{AlrV}$ and $\mathrm{BsrV}$ for various amounts $(1.25-60 \mathrm{mM})$ of L-amino-acid substrates were calculated using data obtained with $0.47 \mu M$ purified racemase (see \$2).

All kinetic constants must be considered as apparent values because of the impossibility of calculating initial enzyme velocities. The results are means \pm $\mathrm{SD}$ of triplicates from two independent experiments. BsrV reaction kinetics for His and Cys were not determined (ND).

\begin{tabular}{clll}
\hline & $K_{\mathrm{m}}(\mathrm{m} M)$ & $k_{\text {cat }}\left(\mathrm{s}^{-1}\right)$ & $k_{\text {cat }} / K_{\mathrm{m}}\left(\mathrm{m}^{-1} \mathrm{~s}^{-1}\right)$ \\
\hline BrsV & & & \\
Ala & $11 \pm 2$ & $1.68 \pm 0.11$ & $15.3 \times 10^{-2}$ \\
Ser & $28 \pm 3$ & $4.68 \pm 0.21$ & $16.7 \times 10^{-2}$ \\
Met & $11 \pm 2$ & $2.94 \pm 0.04$ & $26.7 \times 10^{-2}$ \\
Leu & $30 \pm 7$ & $2.30 \pm 0.28$ & $7.7 \times 10^{-2}$ \\
Cys & $\mathrm{ND}$ & $\mathrm{ND}$ & $\mathrm{ND}$ \\
Gln & $22 \pm 3$ & $2.59 \pm 0.16$ & $11.8 \times 10^{-2}$ \\
Asn & $15 \pm 4$ & $0.05 \pm 0.004$ & $0.3 \times 10^{-2}$ \\
His & $\mathrm{ND}$ & $\mathrm{ND}$ & $\mathrm{ND}$ \\
Lys & $9 \pm 1$ & $4.76 \pm 0.11$ & 52.9 \\
Arg & $18 \pm 3$ & $5.09 \pm 0.11$ & 28.3 \\
AlrV & & & \\
Ala & $1.5 \pm 0.6$ & $1.42 \pm 0.003$ & 94.5 \\
Ser & $17 \pm 8$ & $1.16 \pm 0.0002$ & 6.8 \\
\hline
\end{tabular}

and HPLC analysis was performed (see Supporting Information).

\subsection{Structural determination}

Crystallization of BsrV, an $\mathrm{R}_{173} \mathrm{~N}_{174} / \mathrm{AA}$ point mutant $(\Delta \mathrm{Cl}-$ BsrV), Aeromonas hydrophila Alr3 (a putative Bsr, renamed $\mathrm{Bsr}_{\mathrm{Ah}}$ ) and the putative primary alanine racemase from A. hydrophila $\left(\mathrm{Alr}_{\mathrm{Ah}}\right)$ was performed using a high-throughput NanoDrop ExtY robot (Innovadyne Technologies Inc.) and the commercial Qiagen screens The JCSG+ Suite and The PACT Suite and the Hampton Research screens Index, Crystal Screen and Crystal Screen 2 following the sitting-drop vapourdiffusion method (see Supporting Information). X-ray data collection was performed at a synchrotron-radiation facility on beamlines ID29 and ID14-4 at the ESRF, Grenoble, France or on the X06SA beamline at SLS, Villigen, Switzerland (see Supporting Information). Data sets were collected using a PILATUS 6M or a Q315r ADSC X-ray detector, and were processed using $X D S$ (Kabsch, 2010) and scaled using SCALA (Evans, 2006) from the CCP4 suite (Winn et al., 2011). The structure was solved by the molecular-replacement method with MOLREP (Vagin \& Teplyakov, 2010) from the CCP4 suite and was refined with PHENIX (Adams et al., 2010) and manually improved using Coot (Emsley \& Cowtan, 2004; see Supporting Information). The stereochemistry of the models was verified using MolProbity (Chen et al., 2010).

\subsection{Bioinformatic analyses}

The identification of new putative broad-spectrum racemases and the residues responsible for substrate specificity was performed by searching with BLAST (Altschul et al., 1997) against a nonredundant protein database and filtering, selecting only those mapped onto the UniProt database. The protein data set was aligned using MUSCLE (Edgar, 2004; v.3.8.31). The resulting multiple sequence alignment was filtered using the Jalview (Waterhouse et al., 2009) tool (v.2.7). The identification of specificity-determining positions (SDPs) was carried out using the JDet package (see Supporting Information).

\subsection{Accession numbers}

The atomic coordinates and structure factors for BsrV, $\Delta \mathrm{Cl}$ BsrV mutant, $\mathrm{Bsr}_{\mathrm{Ah}}$ and $\mathrm{Alr}_{\mathrm{Ah}}$ (PDB entries 4beu, 4beq, 4bf5 and 4bhy, respectively) have been deposited in the Protein Data Bank, Research Collaboratory for Structural Bioinformatics, Rutgers University, New Brunswick, New Jersey, USA (http://www.rcsb.org/).

\section{Results}

\subsection{BsrV is a promiscuous paralogue of the $V$. cholerae} alanine racemase AlrV

Comparisons of the substrate specificities of BsrV and AlrV, the presumed alanine-specific racemase from $V$. cholerae, revealed that $\mathrm{BsrV}$ is able to utilize a much broader range of substrates (Figs. $1 b, 1 c$ and $1 d$ ). Unlike AlrV, which only catalyzes the interconversion of L-Ala and L-Ser and their corresponding D forms (Fig. $1 c$ and Supplementary Fig. S1b), BsrV reversibly racemizes ten of the 19 natural chiral amino acids known, including both non- $\beta$-branching aliphatic amino acids (Ala, Leu, Met, Ser, Cys, Gln and Asn) and positively charged amino acids (His, Lys and Arg) (Fig. $1 d$ and Supplementary Figs. S1c and S1d). Additionally, it catalyzes the racemization of several amino acids that are not typically incorporated into proteins (Supplementary Fig. S1e). However, BsrV did not alter the chirality of negatively charged (i.e. Glu and Asp) or aromatic (i.e. Tyr, Trp and Phe) amino acids and displayed minimal activity towards $\beta$-branched aliphatic (i.e. Ile, Val and Thr) potential substrates (Fig. 1d).

\subsection{Reaction kinetics of BsrV and AlrV}

Kinetic analyses, using individual and pooled substrates, were also performed in order to better characterize the activities of BsrV and AlrV. Most analyses were performed using the His-tagged mature proteins used in structural studies because control assays performed with untagged mature BsrV showed that the affinity tag did not alter the activity of the enzyme (Supplementary Fig. S1f). The kinetic analyses revealed that the catalytic efficiency of BrsV (as reflected by $\left.k_{\text {cat }} / K_{\mathrm{m}}\right)$ varies depending upon the amino-acid substrate and suggest that it acts more readily upon lysine, arginine and methionine than upon other substrates (Table 1). Assays using pooled substrates largely confirmed this idea: BsrV incubated with its ten potential L-amino-acid substrates efficiently formed D-Arg and D-Lys, but produced lower amounts of other DAA than when they were assayed singly (Supplementary Figs. S1 $g$ and S1h). Consequently, the high production of D-Met and D-Leu by $V$. cholerae in vivo (the principal products of BrsV; Lam et al., 2009) is likely to reflect the relatively high availability of the corresponding L-amino acids. 
Comparative analyses of $\mathrm{BsrV}$ and AlrV reaction kinetics revealed that the average $k_{\text {cat }}$ of $\mathrm{BsrV}$ is higher than that of AlrV. However, the $K_{\mathrm{m}}$ values for $\mathrm{BsrV}$ were also around tenfold higher than the $K_{\mathrm{m}}$ of $\mathrm{AlrV}$ for alanine, its preferred substrate, and consequently $k_{\text {cat }} / K_{\mathrm{m}}$ for alanine racemization by AlrV is higher than that of BsrV for its substrate amino acids (Table 1). These facts fit well with the proposal that the development of catalytic multispecificity is often accompanied by a reduction in kinetic performance (Babtie et al., 2010; Khersonsky et al., 2006).

\subsection{The crystal structure reveals unique structural features in BsrV}

Purified BsrV was crystallized and its structure was solved at atomic resolution (1.15 ̊; Table 2). As observed for monospecific Alrs, BsrV folds as a globular homodimeric enzyme in which both monomers participate in the elaboration of the active sites (Fig. 2a). Analyses using the $D A L I$ server (Holm \& Rosenström, 2010) indicate that the closest structural homologues of BsrV are alanine racemases from Bacillus anthracis $\left(\mathrm{Alr}_{\mathrm{Bax}}\right.$; Couñago et al., 2009; r.m.sd. of $2.0 \AA$ for $361 \mathrm{C}^{\alpha}$ atoms) and E. coli $\left(\mathrm{Alr}_{\mathrm{Ec}}\right.$; $\mathrm{Wu}$ et al., 2008; r.m.s.d. of $2.5 \AA$ for $347 \mathrm{C}^{\alpha}$ atoms). Since the crystallization of AlrV was unsuccessful, further comparative analyses of BsrV were performed with $\operatorname{Alr}_{\mathrm{Ec}}\left(E\right.$-value of $3 \times 10^{-157}$ to AlrV; Supplementary Fig. S1a). Superimposition of the $\mathrm{BsrV}$ and $\mathrm{Alr}_{\mathrm{Ec}}$ structures readily reveals their extensive similarity; nonetheless, substantial differences are also apparent (Fig. $2 b$ and Supplementary Figs. S2 and S3).

The entry channel to the catalytic site is approximately twofold wider in BsrV than in $\mathrm{Alr}_{\mathrm{Ec}}$ (roughly $10 \times 14 \AA$ in BsrV compared with $7 \times 9.5 \AA$ in $\mathrm{Alr}_{\mathrm{Ec}}$; Fig. $2 b$ and Supplementary Figs. S2 $b$ and S2c). This difference is a direct consequence of shorter loops in the side walls of the catalytic entry site in BsrV and of the different orientation of helix $\alpha 10$ (Fig. $2 b$ and Supplementary Figs. S $2 b$ and $\mathbf{S} 2 c$ ). In addition, the catalytic entry site has a negative charge in BsrV but not in $\mathrm{Alr}_{\mathrm{Ec}}$ (Supplementary Fig. S2d), perhaps impeding the entry of acidic substrates into the catalytic site and thereby contributing to the selectivity of BsrV for neutral and basic amino acids. BsrV also contains a wider tunnel connecting the entry to the catalytic centre (the narrowest width of the BsrV tunnel is $9.0 \AA$ compared with $6.3 \AA$ in $\mathrm{Alr}_{\mathrm{Ec}}$ ).

The structure of $\mathrm{BrsV}$ also differs from that of $\mathrm{Alr}_{\mathrm{Ec}}$ owing to the presence of an additional structural element formed from the sequence at the $\mathrm{N}$-terminus of the protein (residues ${ }^{24} \mathrm{APLHIDT}^{30}$ ). These amino acids fold into a stable, hingelike complementary structure between monomers that shifts their relative positions (Figs. $2 b$ and $2 c$ ). In BsrV monomers, the relative positions of the N-terminal domain (residues 24279) and the C-terminal domain (residues 280-410) are offset by a $9^{\circ}$ rotation compared with the equivalent domains in $\mathrm{Alr}_{\mathrm{Ec}}$ (Fig. 2c), and an average displacement of $1.5 \AA$ for the whole protein backbone is observed. Consequently, there are dramatic differences between the disposition and conformation of the active sites of $\mathrm{BrsV}$ and those of $\mathrm{Alr}_{\mathrm{Ec}}$ (e.g. an increased distance between active sites from $37.6 \AA$ in Alr to $45 \AA$ in BsrV; Fig. $2 c$, right). The N-terminal structure of BrsV appears to be important for the activity of the enzyme, since a single mutation in this structure (P25E; Fig. $2 b$ and Supplementary S3a) completely abolishes the activity of $\mathrm{BrsV}$ towards Ala and Met and dramatically impairs $(70 \%$ reduction) its activity towards Arg.

\subsection{The catalytic machinery of BsrV}

In addition to the differences between the relative positions of protein domains in $\mathrm{BsrV}$ and $\mathrm{Alr}_{\mathrm{Ec}}$, comparative structural analyses revealed significant differences between the catalytic 
centres of the two enzymes. Notably, the enzymes use different means to position their PLP cofactor (Fig. $3 a$ and Supplementary Fig. S3b). In BsrV, a Tyr residue that is conserved
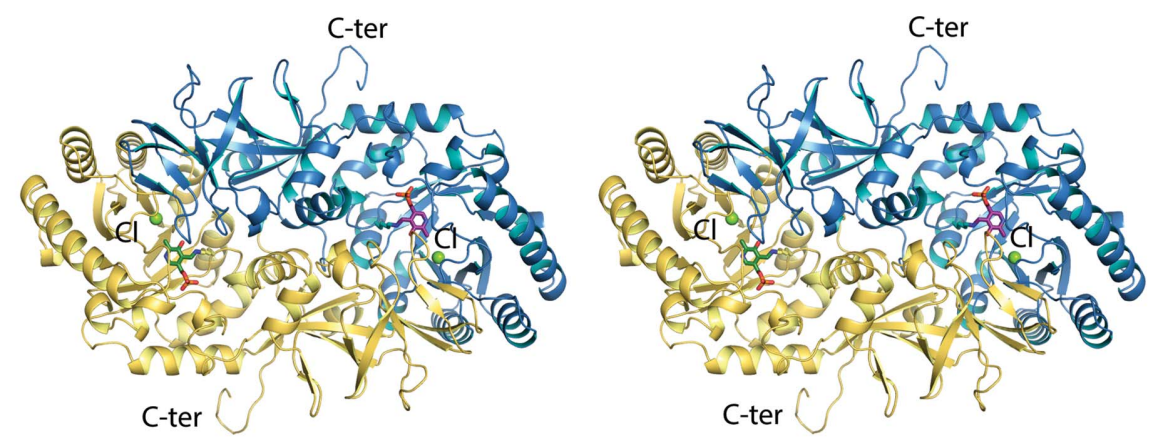

(a)
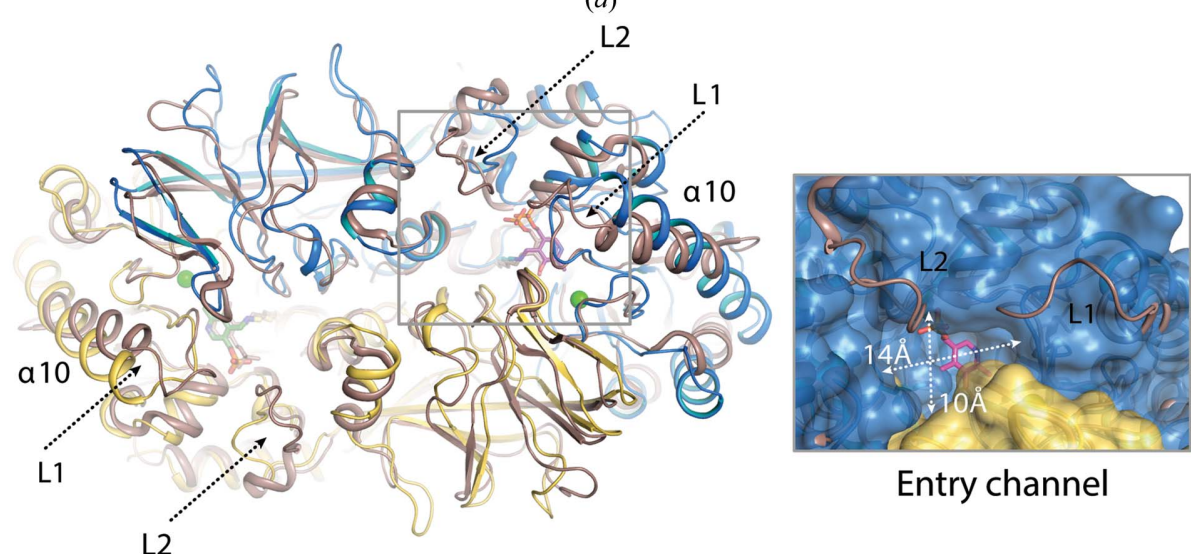

Entry channel

$\mathrm{L} 2$
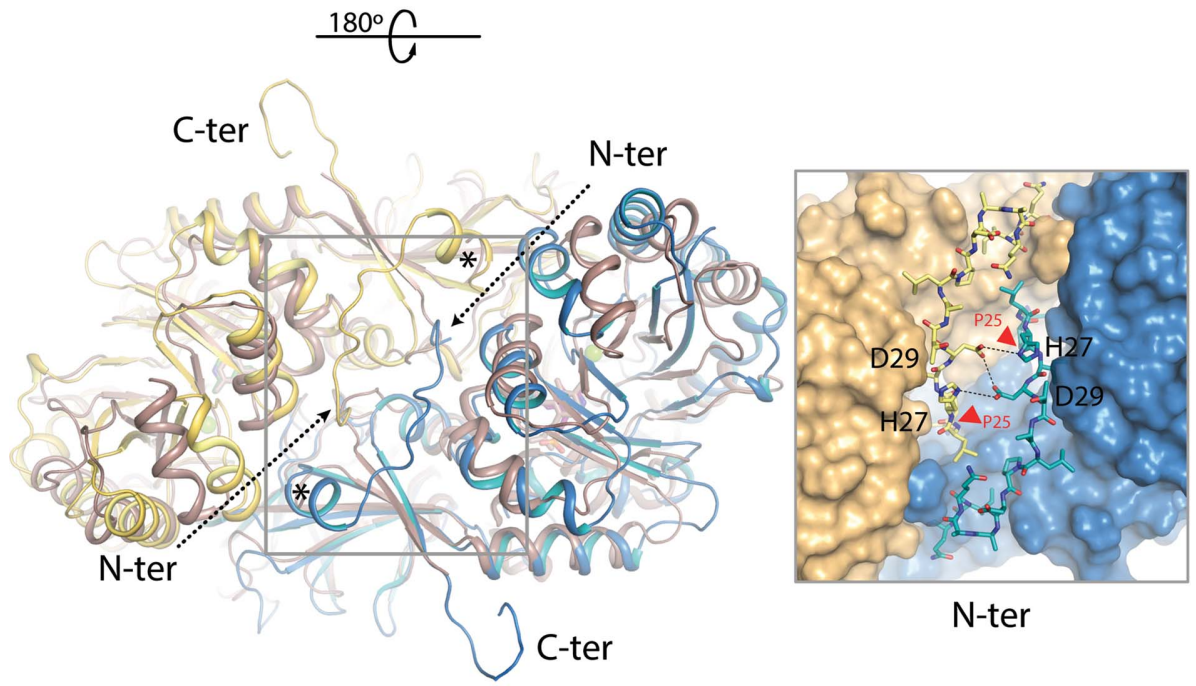

(b)

Figure 2

Three-dimensional structure of the BsrV dimer. (a) Crystal structure of a BsrV dimer with monomers depicted in yellow and blue. PLP molecules are shown in sticks and $\mathrm{Cl}^{-}$ions are shown as green spheres. (b) Structural superimposition of BsrV coloured as in $(a)$ and Ala racemase from E. coli $\left(\mathrm{Alr}_{\mathrm{Ec}}\right)$ in brown. Loops $\mathrm{L} 1$ and $\mathrm{L} 2$ and $\alpha$-helix $\alpha 10$ at the entry channel are labelled [six residues in L1 (204-210) and 11 residues in L2 (267-278) in BstV versus ten residues in L1 (159-169) and 18 residues (216-234) in $\mathrm{L} 2$ in $\left.\mathrm{Alr}_{\mathrm{Ec}}\right]$. On the right, differences in their active-site cavities are shown. White arrows show the dimensions of the entrance of the BsrV cavity. At the bottom of $(b)$, a $180^{\circ}$ rotation view (back side) of the dimer is shown and the BsrV N-terminal insertion is highlighted in the box (bottom right). The position of the $\mathrm{N}$-terminal residue for both monomers in the $\mathrm{Alr}_{\mathrm{Ec}}$ structure is marked by an asterisk. In the close-up view of the back-side region of BsrV, the N-terminal insertion is shown as sticks. Polar interactions between the N-terminal extensions from both monomers are represented as dotted lines. The position of Pro25 is indicated by a red arrow.
N-ter

among Alrs and is typically involved in coordination of the phosphate moiety of PLP is replaced by a proline residue (Pro391), thereby enlarging the space within the active site. Phosphate coordination in BsrV instead depends on Ser245 and Tyr208 and a network of interactions mediated by water molecules (Fig. $3 a$ and Supplementary Fig. S3b). No differences are observed in the stabilization of the pyridoxal ring, where Arg260 stabilizes the $\mathrm{N}$ atom of the ring through its $\mathrm{N}^{\varepsilon}$ atom $(2.82 \AA)$.

The active site of BrsV also lacks an $\mathrm{N}$-carboxylated lysine (Kcx) that in $\mathrm{Alr}_{\mathrm{Ec}}$ and most alanine racemases stabilizes an arginine involved in substrate binding within the active site (Watanabe et al., 2002). In BsrV, Kcx is replaced by Ala165 and the substratebinding $\operatorname{Arg}(\operatorname{Arg} 173)$ is then stabilized in the active site by the presence of an ion (Fig. $3 a$ and Supplementary Figs. $\mathrm{S} 3 b$ and $\mathrm{S} 3 c$ ). Its tetracoordination (with one water molecule at $3.28 \AA$, the $\mathrm{N}^{\delta 2}$ atom of Asn174 at $3.58 \AA$, the $\mathrm{N}^{\varepsilon}$ atom of $\operatorname{Arg} 173$ at $3.67 \AA$ and the $\mathrm{N}^{\eta 2}$ atom of Arg173 at $3.61 \AA$ ) and low $B$ factor suggested that it was chloride $\left(\mathrm{Cl}^{-}\right.$; Fig. $3 a$ and Supplementary S3c). A $\mathrm{Cl}^{-}$ion has also been found in the monospecific alanine racemase $\mathrm{Alr}_{\mathrm{Bax}}$; however, unlike in $\mathrm{BsrV}$, coordination of $\mathrm{Cl}^{-}$in $\mathrm{Alr}_{\mathrm{Bax}}$ is mediated by the same residues that interact with $\mathrm{Kcx}$ in $\mathrm{Alr}_{\mathrm{Ec}}$ and other enzymes that contain Kcx (Couñago et al., 2009). Furthermore, the position occupied by Asn174 in BsrV (Fig. 3a) contains hydrophobic residues (Leu and Ile) in $\mathrm{Alr}_{\mathrm{Ec}}, \mathrm{Alr}_{\mathrm{Bax}}$ and other monospecific Alrs. Collectively, these analyses provide strong evidence that the presence of a negative charge near the catalytic residue is critical for amino-acid racemization by these enzymes; however, they also illuminate the differing catalytic environments in the active sites of enzymes with distinct specificities.

The relevance of the $\mathrm{Cl}^{-}$to $\mathrm{BsrV}$ activity was further investigated by the structural and biochemical characterization of a BsrV $\mathrm{R}_{173} \mathrm{~N}_{174} / \mathrm{AA}$ point mutant $(\Delta \mathrm{Cl}-\mathrm{BsrV})$. As expected, analysis of the crystal structure of $\triangle \mathrm{Cl}-\mathrm{BsrV}$ (Table 2 and Fig. $3 b$ ) revealed that the $\mathrm{Cl}^{-}$was absent. However, the structure of $\Delta \mathrm{Cl}-\mathrm{BsrV}$ 
was otherwise very similar to that of BsrV; the only minor changes observed were in the side chains of Tyr208 and His204 (Fig. $3 b$ ). As anticipated, $\Delta \mathrm{Cl}$-BsrV had a highly reduced or no ability to racemize most amino acids (Fig. $3 c$ and Supplementary Figs. S3d and S3e), consistent with $\mathrm{Cl}^{-}$and $\operatorname{Arg} 173$ serving to replace Kcx in stabilizing a variety of substrates in the active site of BsrV.

\subsection{Identification of molecular signatures that distinguish BsrV-like and Alr-like racemases}

We hypothesized that the basis for the broad substrate tolerance of BsrV might be recognizable through sequence analysis and therefore searched for a molecular footprint of residues that are conserved among BsrV-like enzymes and differ from those found in monospecific alanine racemases. To initiate this analysis, a representative, nonredundant, set of PLP-dependent amino-acid racemase sequences (Alr and Bsr) from different bacteria were collected and aligned. Multiple sequence alignment (MSA; see §2) revealed differential conservation patterns that allowed clustering of proteins into three subfamilies (Fig. 4a). Subfamily 1 (in red) is composed of 84 proteins and includes AlrV, $\mathrm{Alr}_{\mathrm{Ec}}$ and Alr2 (DadB) from E. coli, which have been experimentally confirmed as alaninespecific racemases (Fig. 1c; Faraci \& Walsh, 1988; Lam et al., 2009). Subfamily 2 (in blue) is comprised of 13 proteins,
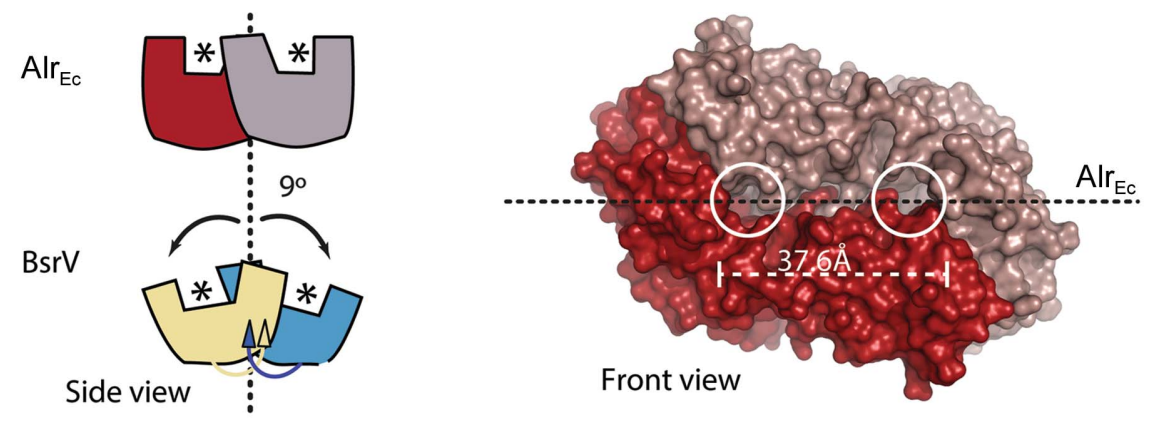

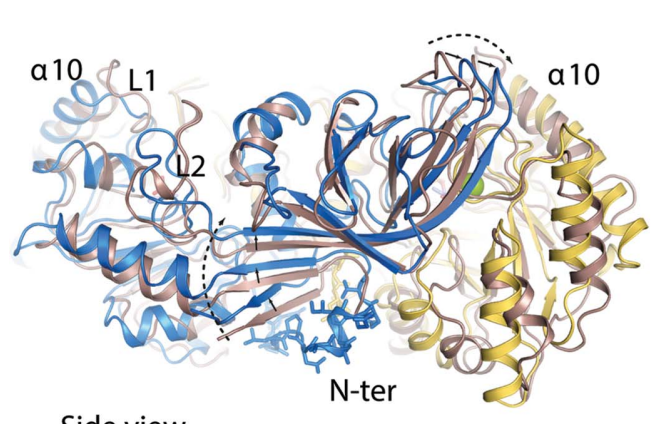

Side view

Figure 2 (continued)

(c)

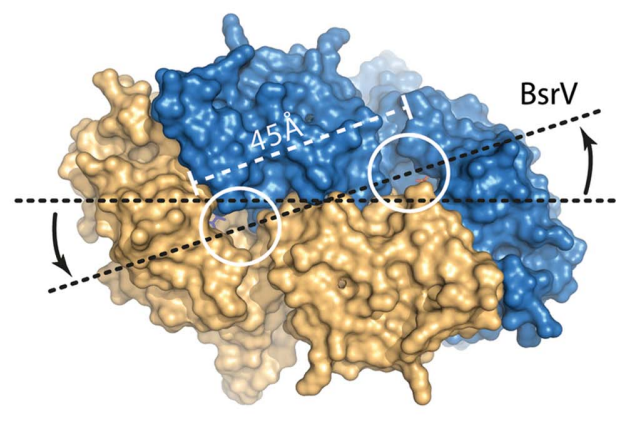

Front view

(c) Different oligomeric arrangement between BsrV and $\mathrm{Alr}_{\mathrm{Ec}}$ and its effect on the spatial distribution of the active sites. A schematic representation of the $\mathrm{Alr}_{\mathrm{Ec}}$ and $\mathrm{BsrV}$ dimers is shown on the left; asterisks mark active sites. A rotation of $9^{\circ}$ between monomers is produced by the $\mathrm{N}$-terminal insertion in BsrV. Bottom left, structural superimposition of BsrV and $\mathrm{Alr}_{\mathrm{Ec}}$ (brown cartoon). Displacement of the BsrV structural core by the $\mathrm{N}$-terminal insertion (sticks) is represented by black arrows. Molecular surfaces for $\mathrm{Alr}_{\mathrm{Ec}}$ and BsrV are shown on the right. The positions of active sites are marked by white circles and differences in distances and position of BsrV active sites versus Alr are highlighted. See also Supplementary Fig. S2. including BsrV (Alr2 from $V$. cholerae). Of the remaining 39 proteins in the data set, 24 proteins were grouped as subfamily 3 (in green). The 15 outlier proteins (in black) could not be assigned to any of the subfamilies (Fig. 4a). Based on the grouping of the characterized racemases, we assumed that the proteins in subfamily 1 are monospecific alanine racemases, while the proteins in subfamily 2 have a broader substrate spectrum. As no member of subfamily 3 or any of the 15 outlier proteins has been biochemically characterized, these proteins were not considered for further sequence analysis.

Further comparative analyses of subfamilies 1 and 2 (see filies. After additional refinement with manual inspecof each potential SDP, this approach allowed the selection enzymes that appear to constitute a (he used to distinguish alanine and (Fig. 4b; discussed further below). 列 BsrV structure revealed that these amino acids affect or reside within features that distinguish BsrV from AlrV, including (A) entry sites (Arg119 and Arg121), the size of (Pro206, Tyr208, Lys216 and Tyr246) and the catalytic machinery (Cys70, Ala165, Asn167, Gly169, Asn174, Gly263, Asn348, Thr349 and Pro391; see Fig. 5h). Notably, specificity determinants were not found within the $\mathrm{N}$-terminal extension of BsrV, although all of the putative broad-spectrum enzymes appear to possess this signature in the mature protein (relative to Alrs) as well as a putative signal sequence (Supplementary Table S3).

An expanded data set containing 2540 putative PLP racemases was similarly grouped into subfamilies and the identities of these families were determined based on the enrichment of proteins described as 'broadspectrum' or 'specific' racemases in the previous analysis (see Supplementary Material). This strategy resulted in the identification of 74 BsrV-like racemases that had probably been misannotated previously as being specific racemases. Intriguingly, most Bsr candidates are found in Gram-negative marine bacteria, primarily $(54.5 \%)$ in members of the Vibrionaceae family (Supplementary Table S4). 


\subsection{The structure of the $A$. hydrophila broad-spectrum racemase mirrors that of BsrV}

To validate our in silico identification of putative broad-spectrum racemases, we performed biochemical and structural characterization of $A$. hydrophila Alr3 (a putative Bsr, renamed $\mathrm{Bsr}_{\mathrm{Ah}}$ ) and the putative primary alanine racemase of $A$. hydrophila $\left(\mathrm{Alr}_{\mathrm{Ah}}\right)$, which were identified from the 'broad-spectrum' and 'specific' subfamilies, respectively (Figs. $4 a$ and $4 c$, Table 2 and Supplementary Fig. S4 and Table S4). As predicted, structural comparison revealed that $\mathrm{Alr}_{\mathrm{Ah}}$ and $\mathrm{Bsr}_{\mathrm{Ah}}$ differ in a manner analogous to that seen for BsrV and $\mathrm{Alr}_{\mathrm{Ec}}$ (Supplementary Fig. S4), and $\mathrm{Bsr}_{\mathrm{Ah}}$ exhibits all of the distinguishing structural features of $\mathrm{BsrV}$ [i.e. $\mathrm{Cl}^{-}$instead of $\mathrm{Kcx}$, a wider entry channel (shorter flanking loops) for the catalytic site, an N-terminal hinge-like extension and conserved signature residues; Fig. $4 c$ and Supplementary Figs. S4d-S $4 h$ ]. $\mathrm{Bsr}_{\mathrm{Ah}}$ also resembles BsrV in its ability to discriminate between amino-acid substrates and displayed catalytic activity against an identical subset of natural amino acids (Supplementary Figs. S4i and S4j). Therefore, the distinct subfamilies of racemases evident in bioinformatic analyses appear to comprise a functionally distinct subset of amino-acid racemases with broad substrate specificity.

\subsection{Molecular modelling of the docking of diverse amino-acid substrates within the BsrV and Alr $_{B s}$ active sites}

To unveil the structural determinants that influence substrate stabilization in specific and broad-range racemases, we took advantage of the crystal structure of $B$. stearothermophilus alanine racemase $\left(\mathrm{Alr}_{\mathrm{Bs}}\right)$ in complex with $\mathrm{N}$-(5'-phosphopyridoxyl)-D-alanine (PLP-D-Ala; Watanabe $e t$ al., 2002; PDB entry 116g) as the reference since this structure mimics the enzymesubstrate catalytic intermediate. We superimposed $\mathrm{Alr}_{\mathrm{Bs}}$ in complex with PLP-D-Ala on BsrV and substituted the alanine analogue by all of the different kinds of amino-acid substrates, selecting the most stable conformation in each case (Figs. 5a-5f). Whilst $\mathrm{Alr}_{\mathrm{Bs}}$ could only accommodate alanine and serine (not shown) in its catalytic site (Figs. $5 b, 5 d$ and $5 f$ and Supplementary Table S $5 b$ ), the BsrV catalytic pocket appears to accommodate all ten amino acids found to be racemized by this enzyme (Figs. $5 a, 5 c$ and $5 e$ ).

Figure 3 Fig. S3.

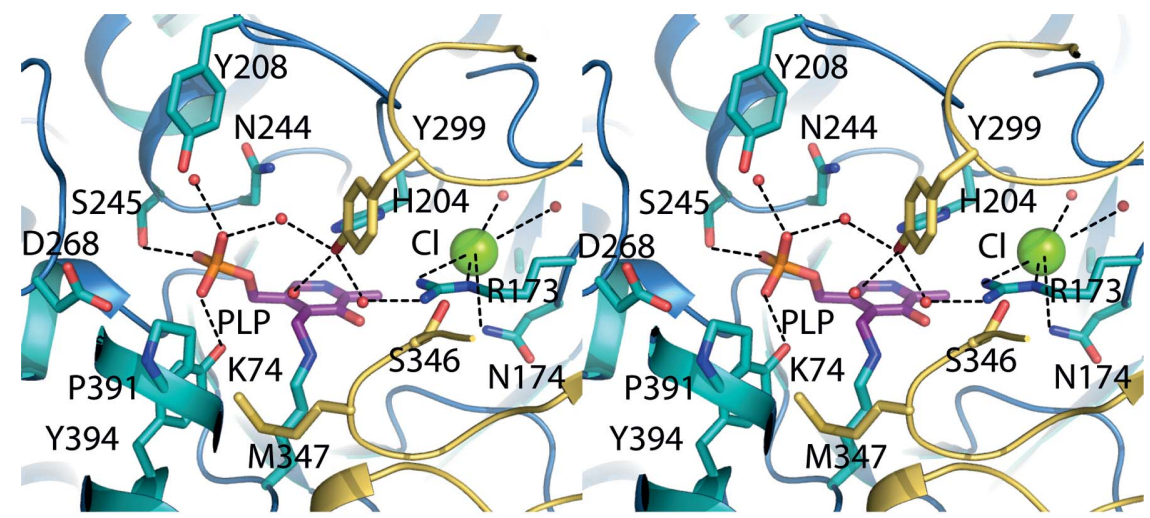

(a)

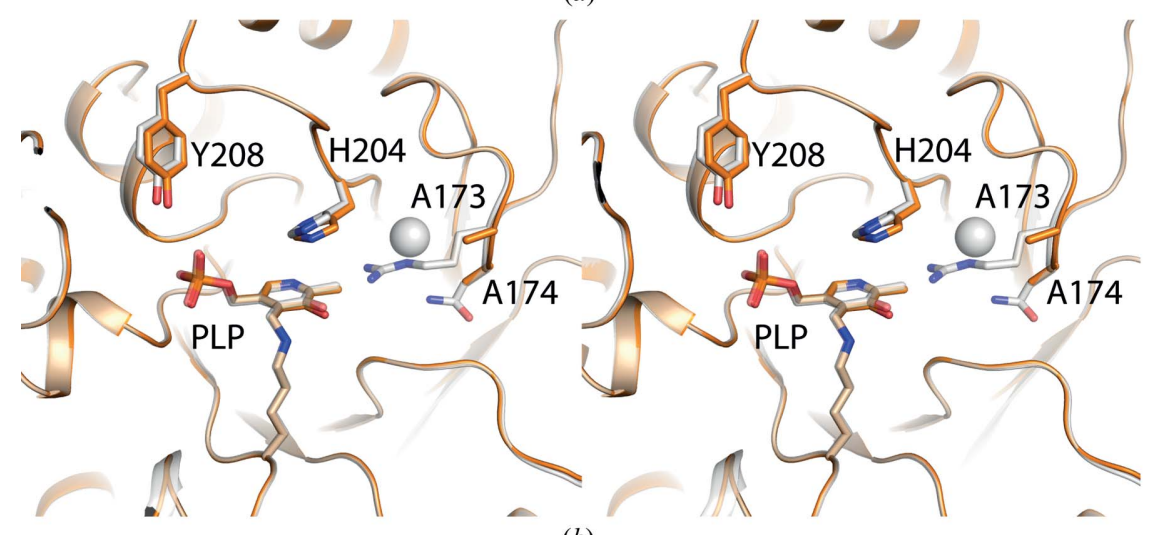

(b)

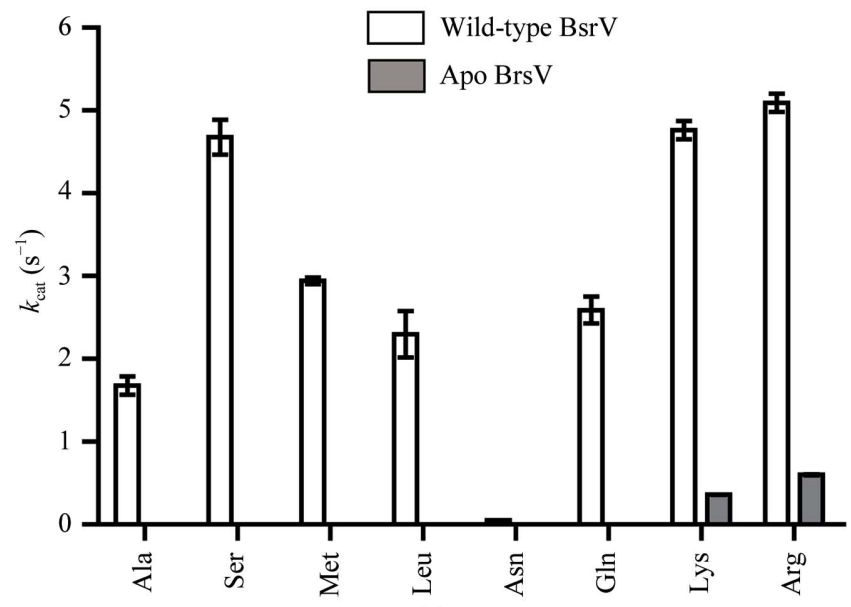

(c)

BsrV and $\Delta \mathrm{Cl}$-BsrV active-site structures and kinetic parameters. (a) Stereoview of the BsrV active site. Relevant residues and PLP are shown as sticks; water molecules and $\mathrm{Cl}^{-}$ions are shown as red and green spheres, respectively. (b) Stereoview showing the structural superimposition of the active sites of wild-type BsrV (shown as a white transparency) and $\Delta \mathrm{Cl}-\mathrm{BsrV}\left(\mathrm{R}_{173} \mathrm{~N}_{174} / \mathrm{AA}\right)$ (coloured orange). No other significant changes apart from the double mutation and consequent $\mathrm{Cl}^{-}$loss (white sphere) are apparent. (c) Comparison between the $k_{\text {cat }}$ of $\Delta \mathrm{Cl}-\mathrm{BsrV}$ and $\mathrm{BsrV}$ for different racemizable amino acids. The results in (c) are means $\pm \mathrm{SD}$ of triplicates from two independent experiments. See also Supplementary

Furthermore, in full agreement with the substrate specificity of BsrV, the remaining nine amino acids present multiple steric clashes when forced to fit into the active site of the enzyme (Supplementary Fig. S5a).

The docking analysis supported two overlapping mechanisms for amino-acid stabilization by BsrV: one for aliphatic 
residues and another for basic amino acids. Aliphatic amino acids (Ala, Ser, Cys, Leu, Gln and Met) are likely to be stabilized in the active site of $\mathrm{BrsV}$ through polar interactions between their carboxylate moiety and Arg173, Tyr299' and Tyr318, in a similar way to that defined for Ala in $\mathrm{Alr}_{\mathrm{Bs}}$ (Figs. $5 a$ and $5 b$ ). In addition, the amino-acid side chains are stabilized through hydrophobic interactions with Met347' in BsrV (Figs. $5 a, 5 c$ and $5 e$ ). Basic amino acids (Arg, Lys and Orn) are stabilized by the same interactions at their carboxylate moieties and, in addition, by hydrogen bonding to Tyr394 and by electrostatic interactions with Asp268 and the PLP phosphate group (Fig. 5e).
Furthermore, docking results neatly explain why BsrV, but not Alrs, racemizes long aliphatic and basic residues. In Alrs, a Tyr residue (Tyr354 in $\mathrm{Alr}_{\mathrm{Bs}}$ ) closes the active-site cavity, which accommodates the short chain of the Ala residue (Fig. $5 b$ ) but produces steric clashes when longer aliphatic, or basic, residues are docked (Figs. $5 d$ and $5 f$ ). The presence of a Pro residue (Pro391) at the equivalent position in BsrV makes sufficient additional space for the proper fitting of both long aliphatic and basic residues (Fig. $5 c$ and 5e).

The differential efficiency of BsrV for its substrates was also in agreement with the docking results. Indeed, the lower activity values found for His and Asn (Supplementary Fig.

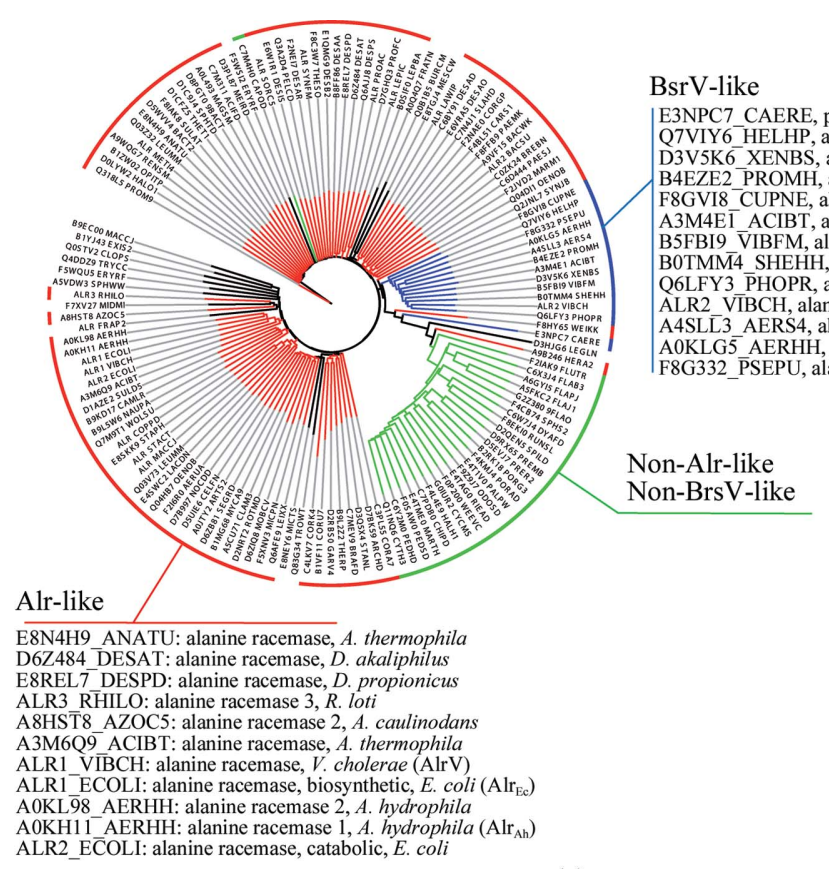

(a)

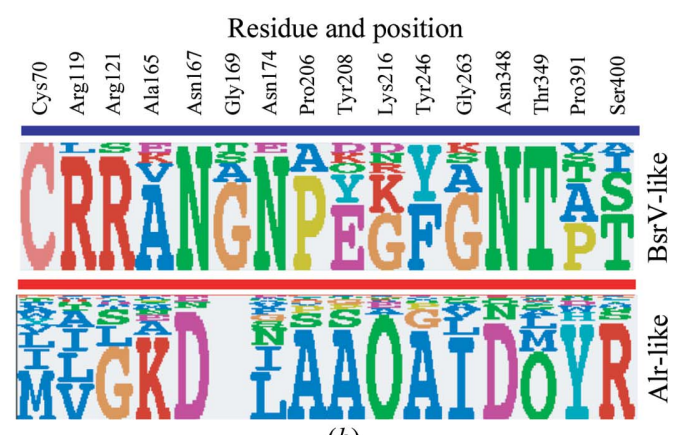

(b)

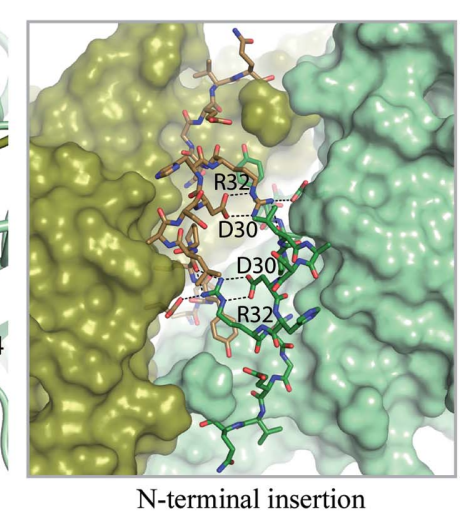

$\mathrm{N}$-terminal insertion
Figure 4

L2

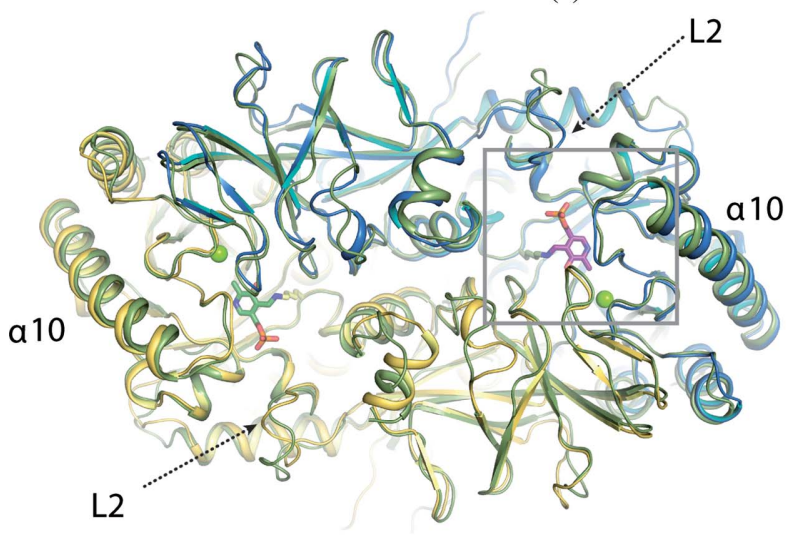

The identification of 16 residues that constitute the molecular footprint for BsrV multispecificity and experimental validation. (a) Tree of BsrV orthologues obtained through bioinformatic sequence analysis (see \$2). Alr-like enzymes are highlighted in red, Bsr-like enzymes in blue and non-Alrlike, non-Bsr-like enzymes in green; 15 outlier proteins that do not group with any of these families are shown in black. All Bsr-like family members (including BsrV) and a small subset of Alr-like enzymes (including AlrV and $\mathrm{Alr}_{\mathrm{Ec}}$ ) are listed. (b) Molecular footprint. Sequence composition (displayed as sequence logos) of the specificity-determining positions for Alr/BsrV-like racemases. The specific residues and their positions are listed at the top. (c) Structural analysis of the broad-spectrum racemase from A. hydrophila $\left(\mathrm{Bsr}_{\mathrm{Ah}}\right)$. A structural superimposition of $\mathrm{BsrV}_{\mathrm{V}}$ with $\mathrm{Bsr}_{\mathrm{Ah}}$ is shown on the left.

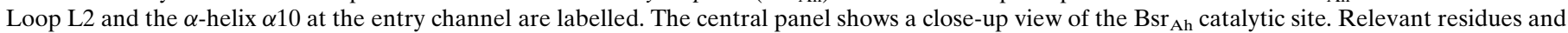
PLP are shown as sticks; water molecules and $\mathrm{Cl}^{-}$ions are shown as red and green spheres, respectively. In the right box, a close-up view of the back-side region $\left(180^{\circ}\right.$ rotation view) of $\mathrm{Bsr}_{\mathrm{Ah}}$ with the $\mathrm{N}$-terminal insertion in stick representation is shown. Polar interactions between relevant residues of the $\mathrm{N}$-terminal extensions from both monomers are represented as dotted lines. See also Supplementary Fig. S4. 


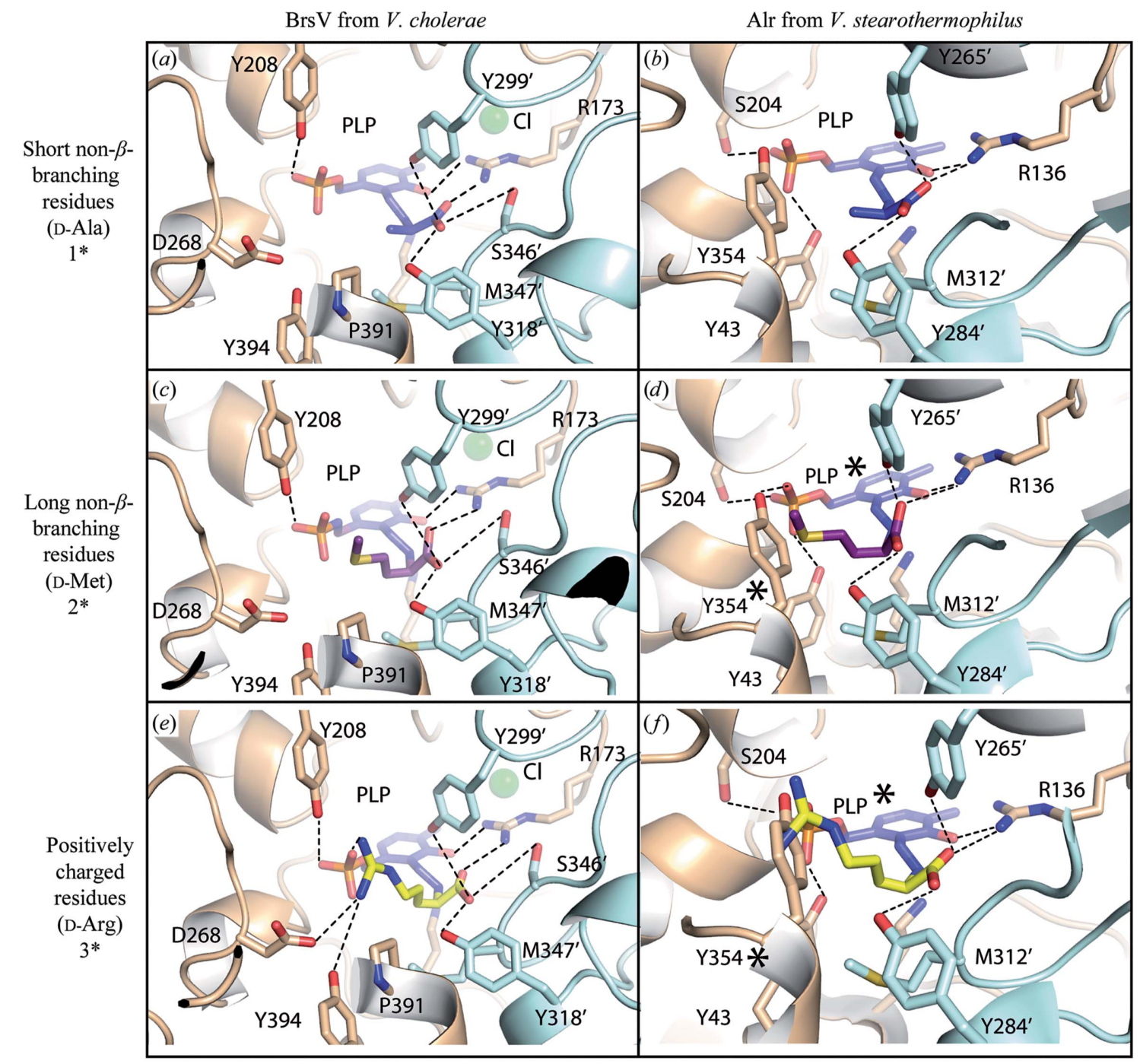

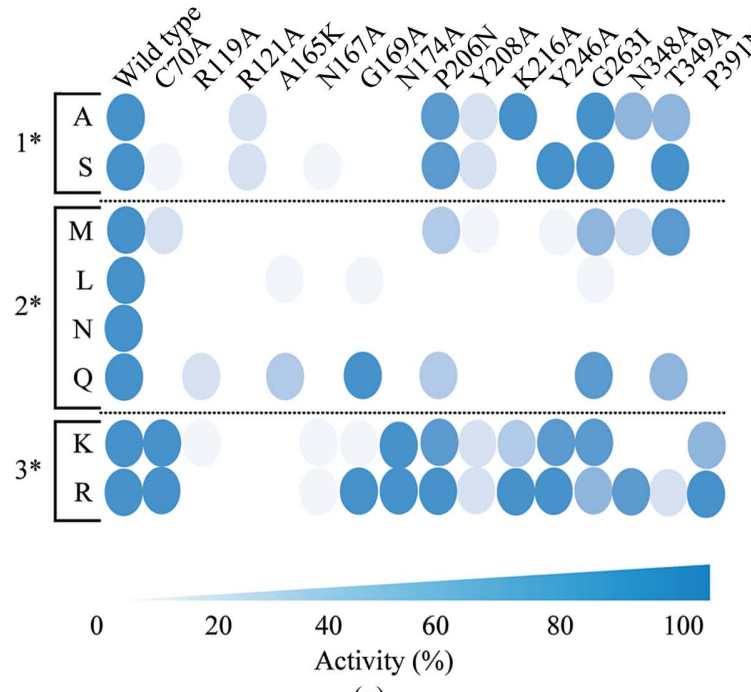

$(g)$

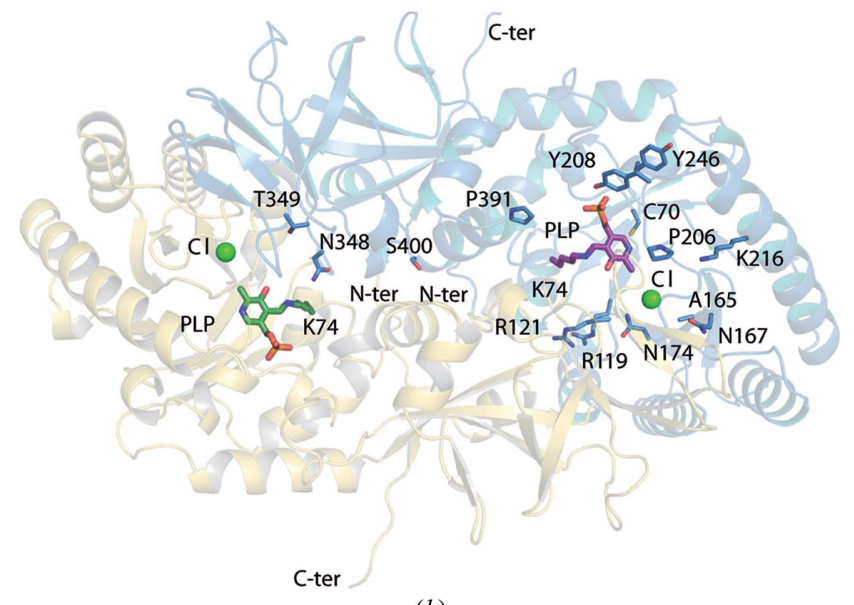

(h)

Figure 5

DAA recognition by BsrV from $V$. cholerae versus Alr from B. stearothermophilus. Docked models of enzyme-substrate catalytic intermediates (BsrV and $\mathrm{Alr}_{\mathrm{Bs}}$ ) for $(a, b)$ short non- $\beta$-branching residues (D-Ala, shown in stick representation with C atoms coloured blue) $\left(1^{*}\right),(c, d)$ long non- $\beta$-branching residues (D-Met, shown in stick representation with $\mathrm{C}$ atoms coloured magenta) $\left(2^{*}\right)$ and $(e, f)$ positively charged residues (D-Arg, shown in stick representation with $\mathrm{C}$ atoms coloured yellow) (3*). Protein residues involved in substrate recognition are labelled and interactions are highlighted as dotted lines. Substrates clashes are labelled by an asterisk. $(g)$ Schematic of specific activity (relative to wild-type BsrV; left column) of BsrV single mutants in the 'signature' SDP. The results correspond to means of triplicates. $(h)$ Location of the SDP within the BrsV structure. See also Supplementary Fig. S5. 
Table 3

Structural analysis of the molecular footprint of BrsV.

\begin{tabular}{|c|c|c|}
\hline Mutation & Protein region & Structural explanation \\
\hline C70A & Active site & Structural stabilization of active site by a disulfide bridge \\
\hline R119A & N-terminal insertion & $\begin{array}{l}\text { Arg119 is involved in salt-bridge interactions allowing } \\
\text { N-terminal insertion in BsrV }\end{array}$ \\
\hline R121A & $\mathrm{N}$-terminal insertion & $\begin{array}{l}\text { Arg121 is involved in salt-bridge interactions allowing } \\
\text { N-terminal insertion in BsrV }\end{array}$ \\
\hline A165K & Active site & $\begin{array}{l}\text { Ala at this position allows the correct stabilization of the } \mathrm{Cl}^{-} \\
\text {ion by Arg173 and Asn174 (in Alrs, this position is } \\
\text { occupied by the } N \text {-carboxylated Lys) }\end{array}$ \\
\hline N167A & Active site & Structural stabilization of loop involved in $\mathrm{Cl}^{-}$coordination \\
\hline G169A & Active site & Loop involved in $\mathrm{Cl}^{-}$coordination \\
\hline N174A & Active site & Residue involved in $\mathrm{Cl}^{-}$coordination \\
\hline $\mathrm{P} 206 \mathrm{~N}$ & Entry loops & $\begin{array}{l}\text { Structural stabilization of the L1 loop (involved in substrate } \\
\text { entry) }\end{array}$ \\
\hline Y208A & Entry loops & Stabilization of the PLP phosphate group \\
\hline $\mathrm{K} 216 \mathrm{~A}$ & Entry loops & Salt-bridge interaction stabilizing $\alpha 10$ at the entry \\
\hline Y246A & Entry loops & Mutation will block movement of the $\mathrm{r} 2$ region \\
\hline G263I & Active site & $\begin{array}{l}\text { Gly residue allows Asp } 268 \text { and Tyr394 stabilization of side } \\
\text { chains of different substrates }\end{array}$ \\
\hline N348A & Active site & Stabilization of loop that includes Met347 at the catalytic site \\
\hline T349A & Active site & \\
\hline P391N & Active site & $\begin{array}{l}\text { This Pro is critical in the broad specificity of BsrV, allowing } \\
\text { the stabilization of side chains from different substrates }\end{array}$ \\
\hline
\end{tabular}

expected, has an especially large effect on the racemization of amino acids with long side chains.

The remaining substitutions have narrower effects, and most frequently limit the activity of BsrV towards long non- $\beta$-branching amino acids, possibly because these substrates encounter more steric obstacles in entering and/or being positioned within the active site. Interestingly, a subset of mutations (G169A, N174A and P391N) that prevent the racemization of non- $\beta$ branching aliphatic amino acids do not markedly impair the activity of BsrV towards basic substrates. Based on the docking models, it seems likely that the side chains of basic substrates are stabilized by different components within the catalytic site (e.g. PLP, Asp268 and Tyr394; Fig. 5e). Analysis of a D268N mutant further confirmed that

S1 $g$ ) can be explained by the limited interactions with Asp268 owing to their shorter side chains (Supplementary Figs. S5c and S5d). Nonetheless, additional elements seem to participate in the stabilization of basic substrates.

\subsection{Functional verification of the putative specificity determinants of BsrV}

To further understand the different specifities of BsrV and AlrV and to assess the significance of each 'signature' residue, we examined the activity of purified BsrV containing individual point mutations at these loci (Figs. $5 g$ and $5 h$ and Supplementary Figs. S $5 f$ and S $5 g$ ). Notably, the mutation of any of the signature sites reduced the activity of BsrV, although the extent of the reduction varied among the mutations and among the classes of substrates (i.e. short and long non- $\beta$-branching aliphatic chains versus positively charged side chains; Fig. $5 g$ and Supplementary S5g). Structural analysis provides likely explanations for the effects of these mutations on BsrV activity (Fig. $5 h$ and Table 3). The analysis of the activity of each BsrV mutant isoform revealed that five substitutions (R119A, R121A, A165K, N167A and Y208A) reduced the activity below $20 \%$ compared with wildtype BsrV for most of the substrates (Fig. $5 \mathrm{~g}$ ). The footprint residues Arg119 and Arg121 are involved in salt-bridge interactions on the back side of the dimer and allow the formation of the $\mathrm{N}$-terminal extension of BsrV. The dramatic effect of their absence confirms our previous conclusion (based on the P25E mutant) that the $\mathrm{N}$-terminal extension is critical for the activity of BsrV towards a variety of substrates. In contrast, the $\mathrm{A} 165 \mathrm{~K}$ and $\mathrm{N} 167 \mathrm{~A}$ substitutions are expected to impair $\mathrm{Cl}^{-}$coordination and consequently the stabilization of the catalytic Arg173. Finally, the Y208A mutant should affect the conformation of the entry loop L1 (Table 3) and, as
Asp268 contributes to the racemization of basic amino acids by BsrV (Supplementary Fig. S5e).

\section{Discussion}

Broad-spectrum amino-acid racemases such as BsrV enable bacteria to generate NCDAA, the varied roles of which in microbial physiology are just beginning to be appreciated (Cava, de Pedro et al., 2011; Kolodkin-Gal et al., 2010, 2012; Lam et al., 2009). Here, we present extensive crystallographic, biochemical and bioinformatic analyses of two broadspectrum racemases ( $\mathrm{BsrV}$ and $\mathrm{Bsr}_{\mathrm{Ah}}$ ) and identify the structural features that enable such enzymes to accommodate a wider range of substrates than related PLP-dependent alanine racemases. We also identify the molecular signature for BsrVlike racemases from diverse microbes that enables them to be distinguished from monospecific alanine racemases.

Although BsrV from $V$. cholerae has previously been reported to produce primarily D-Met and D-Leu (Lam et al., 2009), we found that this enzyme can also racemize eight additional amino acids typically found in proteins, as well as several nonproteinogenic amino acids. The substrates of BrsV fall into two main classes: non- $\beta$-branched aliphatic amino acids (i.e. Ala, Ser, Leu, Cys, Gln, Asn and Met) and basic amino acids (i.e. Arg, Lys and His). Although D forms of the latter class were not detected in vivo, kinetic analyses indicate that they can be readily generated by BsrV, perhaps facilitated by additional stabilizing interactions between these substrates and the active site of BsrV (Figs. 5a, 5c and 5e). Lys and Arg have also been found to be the preferred substrates of a broad-spectrum periplasmic racemase from Pseudomonas taetrolens (Matsui et al., 2009). The enhanced active-site stabilization predicted for basic substrates may account for the resilience of the catalytic activity of mutant BsrV with such substrates relative to large uncharged aliphatic substrates 
(Fig. $5 g$ ). The substrate range of $\mathrm{BsrV}$, which includes activity towards non-natural substrates (e.g. ornithine, norleucine, homoserine, $\mathrm{N}$-acetyl lysine methyl ester, diaminobutyrate and aminobutyrate; Supplementary Fig. S1e), is broader than any other known amino-acid racemase and suggests that it has great potential for biotechnological and industrial applications. Currently, production of DAA is an expensive process that is typically reliant upon inefficient chemical catalysts (Gong et al., 2010; Green et al., 2009; Toth \& Richard, 2007).

Interestingly, all of the Bsr-family racemases are encoded by Gram-negative bacteria. Since some Gram-positive organisms (e.g. B. subtilis) have also been reported to produce NCDAA, this result suggests that additional families of broad-spectrum enzymes may remain to be identified. Consistent with this idea, the spectrum of NCDAA produced by B. subtilis includes several amino acids that cannot be generated by BsrV (e.g. D-Tyr and D-Trp; Kolodkin-Gal et al., 2010). Additional broad-spectrum enzymes may not be related to the PLPdependent racemases such as Alrs and Bsrs, but might instead bear a resemblance to other known racemases, such as MurI.

Comparative structural analyses of BsrV-family broadspectrum racemases $\left(\mathrm{BsrV}\right.$ and $\mathrm{Bsr}_{\mathrm{Ah}}$ ) and more restricted enzymes $\left(\mathrm{Alr}_{\mathrm{Ah}}, \mathrm{Alr}_{\mathrm{Ec}}\right.$ and $\left.\mathrm{Alr}_{\mathrm{Bs}}\right)$ suggest that the catalytic plasticity of these racemases has three essential determinants: the arrangement and features of the entry site, the width of the channel leading to the active site and its catalytic environment, which is able to accommodate and stabilize substrates of diverse chemical natures. There is no indication that the underlying mechanism of racemization differs between the two enzyme subfamilies since the dominant catalytic moeties (catalytic Lys and Tyr) are found in both. The wider entry site and channel of BsrV are likely to facilitate interaction with amino-acid substrates larger than alanine; indeed, structural, biochemical and modelling analyses suggest that the entry channel of BrsV can also accommodate peptide chains which are markedly larger. The catalytic site of BsrV-like racemases is also less constrained than those of related alanine racemases, apparently owing both to differences in the components of the chambers (e.g. $\mathrm{Cl}^{-}$and Pro391) and to different arrangements of the enzymatic domains that interact to form them. Previous analyses have revealed variability in the relative positioning of racemase $\mathrm{N}$ - and $\mathrm{C}$-terminal domains owing to changes in the amino acids at the dimer interface (Couñago et al., 2009). In broad-spectrum racemases, this interface is markedly altered by the presence of a new structure formed from an extended $\mathrm{N}$-terminal sequence. Mutational analyses indicate that the $\mathrm{N}$-terminal extension, as well as the 16 'signature' amino acids that can be used to identify broadspectrum enzymes, are critical for racemase activity on the full set of substrates. Interestingly, mutations in most signature loci did not revert BsrV to an alanine-specific racemase; instead, its activity towards large aliphatic side chains was often lost, while its activity towards basic amino acids was preserved.

Collectively, given the impact of NCDAA on a variety of cellular processes $[$ e.g. biofilm stability, sporulation and cell communication (Anfora et al., 2007; Kolodkin-Gal et al., 2010; O'Connor \& Zusman, 1997)], structural and regulatory characterization of broad-spectrum racemases might open the door to the design of new drugs (Conti et al., 2011) that would challenge the adaptability of pathogenic bacteria to adverse conditions (i.e. during infection).

We thank Stavroula Hatzios for insightful comments on the manuscript and Lourdes Infantes for help in data-mining analysis. Special thanks to F. Lopez-Gallego for providing DAAO. Research in the Cava laboratory is supported by the MINECO, Spain (RYC-2010-06241), Universidad Autonoma de Madrid (UAM-38) and by the Knut and Alice Wallenberg Foundation (KAW). Additionally, this work was supported by the BFU2011-25326 MEC grant (JAH), by the S2010/BMD2457 grant from CAM (JAH) and by HHMI (MKW).

\section{References}

Adams, P. D. et al. (2010). Acta Cryst. D66, 213-221.

Altschul, S. F., Madden, T. L., Schäffer, A. A., Zhang, J., Zhang, Z., Miller, W. \& Lipman, D. J. (1997). Nucleic Acids Res. 25, 3389-3402.

Anfora, A. T., Haugen, B. J., Roesch, P., Redford, P. \& Welch, R. A. (2007). Infect. Immun. 75, 5298-5304.

Austin, B. (2010). Vet. Microbiol. 140, 310-317.

Babtie, A., Tokuriki, N. \& Hollfelder, F. (2010). Curr. Opin. Chem. Biol. 14, 200-207.

Bricogne, G., Blanc, E., Brandl, M., Flensburg, C., Keller, P., Paciorek, W., Roversi, P., Smart, O. S., Vonrhein, C. \& Womack, T. O. (2011). BUSTER. Cambridge: Global Phasing Ltd.

Cava, F., de Pedro, M. A., Lam, H., Davis, B. M. \& Waldor, M. K. (2011). EMBO J. 30, 3442-3453.

Cava, F., Lam, H., de Pedro, M. A. \& Waldor, M. K. (2011). Cell. Mol. Life Sci. 68, 817-831.

Chang, C. Y., Thompson, H., Rodman, N., Bylander, J. \& Thomas, J. (1997). Ann. Clin. Lab. Sci. 27, 254-259.

Chen, C. (1996). Oral Microbiol. Immunol. 11, 425-427.

Chen, V. B., Arendall, W. B., Headd, J. J., Keedy, D. A., Immormino, R. M., Kapral, G. J., Murray, L. W., Richardson, J. S. \& Richardson, D. C. (2010). Acta Cryst. D66, 12-21.

Chen, Y., Zhang, W., Shi, Q., Hesek, D., Lee, M., Mobashery, S. \& Shoichet, B. K. (2009). J. Am. Chem. Soc. 131, 14345-14354.

Conti, P., Tamborini, L., Pinto, A., Blondel, A., Minoprio, P., Mozzarelli, A. \& De Micheli, C. (2011). Chem. Rev. 111, 6919-6946.

Couñago, R. M., Davlieva, M., Strych, U., Hill, R. E. \& Krause, K. L. (2009). BMC Struct. Biol. 9, 53.

Levie, R. de (2001). How to Use Excel in Analytical Chemistry and in General Scientific Data Analysis. Cambridge University Press.

Delong, E. F., Franks, D. G. \& Yayanos, A. A. (1997). Appl. Environ. Microbiol. 63, 2105-2108.

Edgar, R. C. (2004). Nucleic Acids Res. 32, 1792-1797.

El-Hajj, Z. W., Allcock, D., Tryfona, T., Lauro, F. M., Sawyer, L., Bartlett, D. H. \& Ferguson, G. P. (2010). Ann. N. Y. Acad. Sci. 1189, 143-148.

Emsley, P. \& Cowtan, K. (2004). Acta Cryst. D60, 2126-2132.

Evans, P. (2006). Acta Cryst. D62, 72-82.

Faraci, W. S. \& Walsh, C. T. (1988). Biochemistry, 27, 3267-3276.

Gong, L., Mulcahy, S. P., Devarajan, D., Harms, K., Frenking, G. \& Meggers, E. (2010). Inorg. Chem. 49, 7692-7699.

Green, B. A., Yu, R. J. \& Van Scott, E. J. (2009). Clin. Dermatol. 27, 495-501.

Hanahan, D. (1985). DNA Cloning: A Practical Approach, Vol. 1, edited by D. M. Glover, pp. 109-135. Oxford: IRL Press.

Harris, J. B., LaRocque, R. C., Qadri, F., Ryan, E. T. \& Calderwood, S. B. (2012). Lancet, 379, 2466-2476.

Heidelberg, J. F. et al. (2000). Nature (London), 406, 477-483.

Herbert, E. E. \& Goodrich-Blair, H. (2007). Nature Rev. Microbiol. 5, 634-646. 
Holm, L. \& Rosenström, P. (2010). Nucleic Acids Res. 38, W545W549.

Horcajo, P., de Pedro, M. A. \& Cava, F. (2012). Microb. Drug Resist. 18, 306-313.

Howard, A., O'Donoghue, M., Feeney, A. \& Sleator, R. D. (2012). Virulence, 3, 243-250.

Inoue, H., Nojima, H. \& Okayama, H. (1990). Gene, 96, 23-28.

Jacobsen, S. M. \& Shirtliff, M. E. (2011). Virulence, 2, 460-465.

Kaasch, A. J., Dinter, J., Goeser, T., Plum, G. \& Seifert, H. (2012). Infection, 40, 185-190.

Kabsch, W. (1993). J. Appl. Cryst. 26, 795-800.

Kabsch, W. (2010). Acta Cryst. D66, 125-132.

Khersonsky, O., Roodveldt, C. \& Tawfik, D. S. (2006). Curr. Opin. Chem. Biol. 10, 498-508.

Kim, S. E., Park, S. H., Park, H.-B., Park, K.-H., Kim, S.-H., Jung, S.-I., Shin, J.-H., Jang, H.-C. \& Kang, S. J. (2012). Chonnam Med. J. 48, 91-95.

Kino, K., Sato, M., Yoneyama, M. \& Kirimura, K. (2007). Appl. Microbiol. Biotechnol. 73, 1299-1305.

Kolodkin-Gal, I., Cao, S., Chai, L., Böttcher, T., Kolter, R., Clardy, J. \& Losick, R. (2012). Cell, 149, 684-692.

Kolodkin-Gal, I., Romero, D., Cao, S., Clardy, J., Kolter, R. \& Losick, R. (2010). Science, 328, 627-629.

Komarova, N. V., Golubev, I. V., Khoronenkova, S. V., Chubar', T. A. \& Tishkov, V. I. (2012). Biochemistry, 77, 1181-1189.

Laemmli, U. K. \& Favre, M. (1973). J. Mol. Biol. 80, 575-599.

Lam, H., Oh, D.-C., Cava, F., Takacs, C. N., Clardy, J., de Pedro, M. A. \& Waldor, M. K. (2009). Science, 325, 1552-1555.

LeMagueres, P., Im, H., Dvorak, A., Strych, U., Benedik, M. \& Krause, K. L. (2003). Biochemistry, 42, 14752-14761.

LeMagueres, P., Im, H., Ebalunode, J., Strych, U., Benedik, M. J., Briggs, J. M., Kohn, H. \& Krause, K. L. (2005). Biochemistry, 44, 1471-1481.

Lenk, S., Moraru, C., Hahnke, S., Arnds, J., Richter, M., Kube, M., Reinhardt, R., Brinkhoff, T., Harder, J., Amann, R. \& Mussmann, M. (2012). ISME J. 6, 2178-2187.

Long, F., Vagin, A. A., Young, P. \& Murshudov, G. N. (2008). Acta Cryst. D64, 125-132.

Matsui, D. \& Oikawa, T. (2010). Chem. Biodivers. 7, 15911602.

Matsui, D., Oikawa, T., Arakawa, N., Osumi, S., Lausberg, F., Stäbler, N., Freudl, R. \& Eggeling, L. (2009). Appl. Microbiol. Biotechnol. 83, 1045-1054.

Murakami, K. (2012). J. Gastroenterol. 47, 724-725.

Nelson, M. D. \& Fitch, D. H. (2011). Methods Mol. Biol. 772, 459470.
Noda, M., Matoba, Y., Kumagai, T. \& Sugiyama, M. (2004). J. Biol. Chem. 279, 46153-46161.

O'Connor, K. A. \& Zusman, D. R. (1997). Mol. Microbiol. 24, 839-850.

O'Hara, C. M., Brenner, F. W. \& Miller, J. M. (2000). Clin. Microbiol. Rev. 13, 534-546.

Pepperell, C., Kus, J. V., Gardam, M. A., Humar, A. \& Burrows, L. L. (2002). Antimicrob. Agents Chemother. 46, 3555-3560.

Pinhassi, J., Pujalte, M. J., Macián, M. C., Lekunberri, I., González, J. M., Pedrós-Alió, C. \& Arahal, D. R. (2007). Int. J. Syst. Evol. Microbiol. 57, 2370-2375.

Richard, J. P., Amyes, T. L., Crugeiras, J. \& Rios, A. (2009). Curr. Opin. Chem. Biol. 13, 475-483.

Rømer Villumsen, K., Dalsgaard, I., Holten-Andersen, L. \& Raida, M. K. (2012). PLoS One, 7, e46733.

Rosenberg, A. H., Lade, B. N., Chui, D.-S., Lin, S.-W., Dunn, J. J. \& Studier, F. W. (1987). Gene, 56, 125-135.

Rózalski, A., Kwil, I., Torzewska, A., Baranowska, M. \& Staczek, P. (2007). Postepy Hig. Med. Dosw. (Online), 61, 204-219.

Seifert, H., Strate, A., Schulze, A. \& Pulverer, G. (1993). Clin. Infect. Dis. 17, 632-636.

Shaw, J. P., Petsko, G. A. \& Ringe, D. (1997). Biochemistry, 36, 1329 1342.

Strych, U. \& Benedik, M. J. (2002). J. Bacteriol. 184, 4321-4325.

Su, Y.-C. \& Liu, C. (2007). Food Microbiol. 24, 549-558.

Sugar, D. R., Murfin, K. E., Chaston, J. M., Andersen, A. W., Richards, G. R., deLéon, L., Baum, J. A., Clinton, W. P., Forst, S., Goldman, B. S., Krasomil-Osterfeld, K. C., Slater, S., Stock, S. P. \& Goodrich-Blair, H. (2012). Environ. Microbiol. 14, 924-939.

Timoney, P. J. (1996). Comp. Immunol. Microbiol. Infect. Dis. 19, 199-204.

Toth, K. \& Richard, J. P. (2007). J. Am. Chem. Soc. 129, 3013-3021.

Vagin, A. \& Teplyakov, A. (2010). Acta Cryst. D66, 22-25.

Vollmer, W., Blanot, D. \& de Pedro, M. A. (2008). FEMS Microbiol. Rev. 32, 149-167.

Watanabe, A., Yoshimura, T., Mikami, B., Hayashi, H., Kagamiyama, H. \& Esaki, N. (2002). J. Biol. Chem. 277, 19166-19172.

Williamson, E. D. \& Oyston, P. C. (2012). J. Med. Microbiol. 61, 911-918.

Waterhouse, A. M., Procter, J. B., Martin, D. M. A., Clamp, M. \& Barton, G. J. (2009). Bioinformatics, 25, 1189-1191.

Winn, M. D. et al. (2011). Acta Cryst. D67, 235-242.

Wu, D., Hu, T., Zhang, L., Chen, J., Du, J., Ding, J., Jiang, H. \& Shen, X. (2008). Protein Sci. 17, 1066-1076.

Zhao, J.-S., Manno, D., Leggiadro, C., O’Neil, D. \& Hawari, J. (2006). Int. J. Syst. Evol. Microbiol. 56, 205-212. 